\title{
MONTE Python for Deep Space Navigation
}

\author{
Jonathon Smith, William Taber, Theodore Drain, Scott Evans, James Evans, Michelle Guevara, William Schulze, \\ Richard Sunseri, Hsi-Cheng $\mathrm{Wu}^{\text {‡** }}$
}

https://youtu.be/E3RhKKpm4TM

\begin{abstract}
The Mission Analysis, Operations, and Navigation Toolkit Environment (MONTE) is the Jet Propulsion Laboratory's (JPL) signature astrodynamic computing platform. It was built to support JPL's deep space exploration program, and has been used to fly robotic spacecraft to Mars, Jupiter, Saturn, Ceres, and many solar system small bodies. At its core, MONTE consists of lowlevel astrodynamic libraries that are written in $\mathrm{C}++$ and presented to the end user as an importable Python language module. These libraries form the basis on which Python-language applications are built for specific astrodynamic applications, such as trajectory design and optimization, orbit determination, flight path control, and more. The first half of this paper gives context to the MONTE project by outlining its history, the field of deep space navigation and where MONTE fits into the current Python landscape. The second half gives an overview of the main MONTE libraries and provides a narrative example of how it can be used for astrodynamic analysis. For information on licensing MONTE and getting a copy visit montepy.jpl.nasa.gov or email mdn_software@jpl.nasa.gov.
\end{abstract}

Index Terms-astrodynamics, aerospace, orbit, trajectory, JPL, NASA

\section{History}

The United States began its reconnaissance of the solar system in the early 1960s. As NASA developed new technologies to build and operate robotic probes in deep space, JPL was working out how to guide those probes to their destinations. In order to fly spacecraft to Mars or Jupiter, engineers needed a way to model their trajectories through interplanetary space. This was partly a problem of astrodynamics, a field of study that mathematically describes how man-made objects move through space. It was also a problem of computation because engineers needed a way to solve these complex astrodynamic equations for real spacecraft. Beyond modeling the motion of spacecraft, engineers needed a way to measure the location of spacecraft over time so they could make informed corrections to their models. They also needed a way of designing engine burns, or maneuvers, that would nudge a wayward probe back on course.

These efforts, collectively known as deep space navigation, quickly became coupled with software and computing. The first programs JPL wrote to navigate spacecraft were written on punchcards and processed through an IBM 7090 mainframe. [Eke05] Advances in computing technology were eagerly consumed by navigators, as more storage and faster processing meant the

* Corresponding author: jonathon.j.smith@jpl.nasa.gov

+ Jet Propulsion Laboratory, California Institute of Technology / NASA

Copyright $\odot 2016$ California Institute of Technology. Government sponsorship acknowledged. This is an open-access article distributed under the terms of the Creative Commons Attribution License, which permits unrestricted use, distribution, and reproduction in any medium, provided the original author and source are credited. models used to fly spacecraft could be made increasingly detailed and sophisticated.

Starting in 1964, a group of engineers, led by Ted Moyer, began developing the astrodynamic algorithms and software that would eventually become the Double Precision Trajectory and Orbit Determination Program, or DPTRAJ/ODP ([Moy71], [Moy03]). Over its forty-plus years of active life, JPL engineers used the DPTRAJ/ODP to navigate the "Golden Age" of deep space exploration. This included the later Mariner and Pioneer missions, Viking, Voyager, Magellan, Galileo, Cassini and more. Also over this time, its base language moved through Fortran IV, Fortran V, Fortran 77 and Fortran 95 as the computational appetites of navigators grew ever larger.

By 1998 it was clear that the aging DPTRAJ/ODP needed to be updated once again. Rather than initiate another refactor, JPL's navigation section commissioned a new effort that would depart from its predecessor in two important ways. First, the new software would be an object-oriented library, written in $\mathrm{C}++$ and exposed to the user as a Python-language library. Second, it would be a general-purpose astrodynamic computing platform, not a dedicated navigation program like the DPTRAJ/ODP. The goal was to create a single library that could be used for astrodynamic research, space mission design, planetary science, etc., in addition to deep space navigation. This new project was affectionately named the Mission Analysis, Operations, and Navigation Toolkit Environment, or MONTE-Python for short.

Throughout the first half of the 2000s, MONTE was carefully constructed by reshaping the algorithms under-pinning the DPTRAJ/ODP into a rigorously tested and well documented object-oriented software package. In 2007, MONTE had its first operational assignment navigating NASA's Phoenix lander to a successful encoutner with Mars. Since 2012, MONTE has powered all flight navigation services at JPL, including the Cassini extended mission, Mars Science Laboratory, MAVEN, GRAIL, Dawn, Mars Reconnaissance Orbiter, Juno, and more. [Eva16]

\section{Deep Space Navigation}

At JPL, the practice of navigating robotic probes in deep space is broken down into three interrelated disciplines: (1) designing a reference trajectory which describes the planned flight path of the spacecraft (mission design), (2) keeping track of the spacecraft position while the mission is in flight (orbit determination), and (3) designing maneuvers to bring the spacecraft back to the reference trajectory when it has strayed (flight path control, Figure 1).

The process of designing a spacecraft reference trajectory begins at the earliest stages of mission planning. Navigators work 


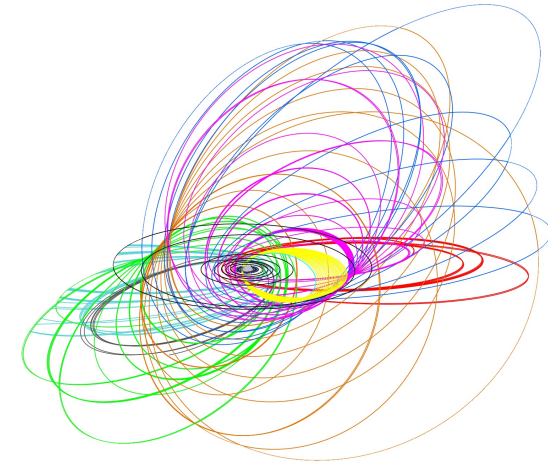

Fig. 1: Illustration of Cassini's reference trajectory at Saturn. The mission designers built this trajectory, and the orbit determination and maneuver design teams keep the spacecraft flying on these orbits during the mission.

closely with mission science teams to put together a reference orbit that allows the spacecraft to take all the desired science measurements. They also work with mission planners and spacecraft system engineers to make sure that the spacecraft is able to withstand the rigors of its planned trajectory. Through a process of increasingly detailed iterations, a process which often takes years, the mission reference trajectory is produced. This reference trajectory serves as the flight plan for the spacecraft. It will be up to the orbit determination and flight path control teams to make sure the spacecraft follows this flight plan when the spacecraft finally launches.

The job of the orbit determination team is to keep track of where the spacecraft has been (orbit reconstruction), where it is currently (orbit determination), and where it will go in the future (orbit prediction). The spacecraft is always drifting away from its planned flight path because of small disturbances it encounters in space. Even the slight pressure of sunlight on the spacecraft can add up over time and push a mission off course. The trajectory designers do their best to account for these disturbances when creating the reference orbit, but there is no accounting for the randomness and unpredictability of the real world. To further complicate matters, once the spacecraft leaves the launch-pad, it can no longer be directly observed. Orbit determination analysts must process various forms of tracking data that are tied mathematically to the evolution of the spacecraft orbit to determine its position at any given time.

Once the orbit determination team has a good estimate for the current location of the spacecraft, the flight path control team is responsible for evaluating how far the spacecraft has drifted from the reference trajectory and designing a maneuver to get the spacecraft back on course. The result of this maneuver design is a $\Delta V$ vector, which stands for delta-velocity or change in velocity. This $\Delta V$ vector represents the direction and magnitude of the required change in the spacecraft velocity which must be accomplished to get the spacecraft back on course. Once in hand, this $\Delta V$ vector will be sent to the spacecraft propulsion team, who will decompose it into thruster firings on the spacecraft. These will be uplinked to the spacecraft, which will then perform the maneuver.

After a maneuver has been performed, the cycle repeats. Perhaps the thrusters were slightly misaligned or the engine cutoff was a second too late. The orbit determination team must examine more tracking data to find out. This iterative relationship between orbit determination and flight path control continues without pause through the lifetime of a flight mission. The spacecraft is constantly wandering off, and must be patiently brought back on course.

\section{MONTE as a Platform}

As previously mentioned, MONTE was built to be a general purpose astrodynamic computing platform, not a dedicated navigation application. It supplies the models and computational algorithms needed for trajectory design, orbit determination and flight path control but doesn't force the end-user into any specific workflow or interface. As a result, before MONTE can be used on a flight mission, it must be deployed for that mission. This entails using MONTE in cooperation with other applications and libraries to assemble a custom navigation framework.

The process of deploying MONTE for a flight mission can be quite involved. The effort to build a navigation system for the Cassini Extended Mission took over two years, and required the use of many other Python libraries in addition to MONTE. The resulting navigation framework can not be properly characterized as MONTE itself. Rather, it is a custom application built using the MONTE library to perform navigation for that specific mission.

This is important to note because it illustrates the way in which MONTE is likely to be useful to those outside JPL. Deep space navigation is (not yet at least) a high-demand field. The majority of astrodynamic computing occurs in other contexts such as Earth-centered navigation (weather and communication satellites, etc), collision avoidance analysis (making sure two spacecraft don't collide), cooperative rendezvous (docking a cargo-ship to the International Space Station) and non-cooperative rendezvous (capturing a malfunctioning satellite), etc. Much the same way that MONTE can be configured and deployed for deep space navigation, it can also be brought to bear on these and other problems across the aerospace industry.

MONTE provides a solid foundation of core systems that make it attractive as a general purpose astrodynamic platform. These include models for trajectories and trajectory queries, coordinate frames and rotations, high-precision time, astrodynamic event searches, numerical integrators, configurable optimizers, and many more. By starting with MONTE, a user can focus on solving the problem at hand, and leave the important-butincidental infrastructure to MONTE.

\section{MONTE and the Python Ecosystem}

MONTE has a decidedly friendly stance when it comes to working with other libraries in the Python scientific computing stack. It makes heavy use of many open-source Python libraries such as matplotlib and IPython (Jupyter), and reciprocally tries to make it easy for users of these systems to interface with MONTE. Many of MONTE's classes can transform themselves into NumPy data types --- a common pattern is for MONTE classes to have a .toArray method which returns a numpy . ndarray. Additionally, the MONTE team has a history of collaboration with matplotlib dating all the way back to the early 2000s. They have contributed code that makes matplotlib able to natively plot MONTE's unit and time systems, and have also open-sourced a custom matplotlib styling-system (github.com/nasa/mplStyle) developed in house.

The MONTE project started in 1998 at a time when the Python language was still relatively new. As a result, MONTE has several 
custom systems that are redundant in the current Python landscape. For instance, MONTE developed an interactive shell similar to IPython and has several numerical computing classes that would generally be dispatched to NumPy in a brand new project.

Historical quirks aside, MONTE considers itself a member of the Python scientific programming community and aims to integrate as seamlessly as possible with other Python libraries. It can be embedded in custom GUI applications, run on a backend server, executed in parallel across a cluster of nodes, and pretty much anything else you would expect of a dynamic, well constructed Python library.

\section{Library Overview}

Most of the functionality of MONTE is encapsulated in the Monte and mpy libraries. Monte is written in C++ and wrapped in Python. It is presented to the end user as a normal, importable Python-language module. The mpy module is written entirely in Python and contains higher level applications built using Monte and other Python libraries.

Convention is to import the main Monte library as M. Throughout this paper, if a class is referred to with the prefix M., it means this class belongs to the main MONTE library (e.g. M.TrajLeg, M. Gm, etc). The following example shows a simple script using the Monte and mpy libraries to get the state of the Cassini spacecraft with respect to Saturn at the time of its Saturn Orbit Insertion (SOI) burn. ${ }^{12}$

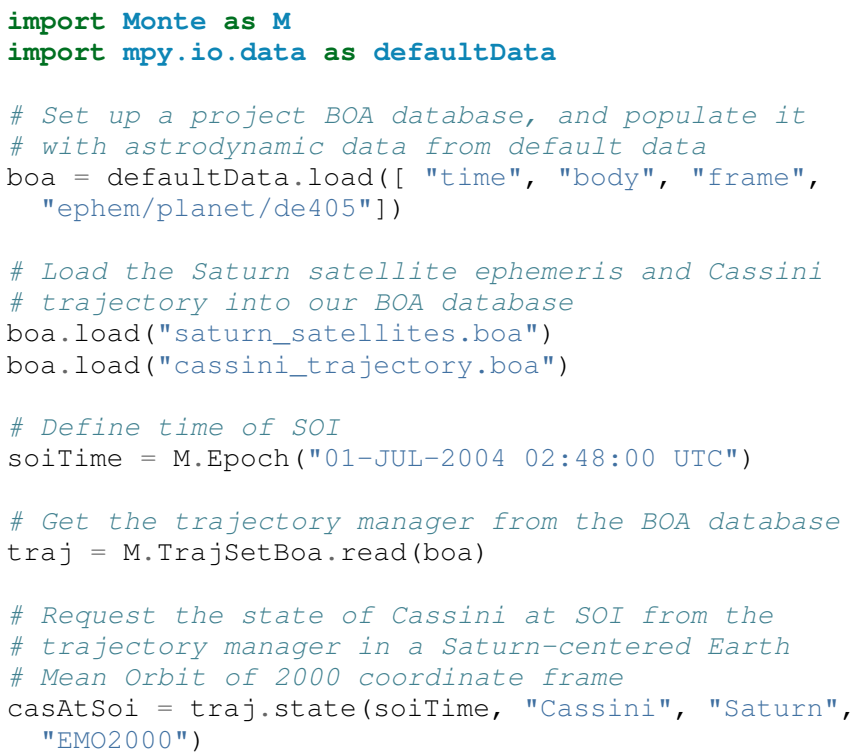

Several of MONTE's core systems --- the basic astrodynamic scaffolding that supports its more advanced functionality --- are used in the above example. These are explained in a short tour of MONTE below.

\section{$B O A$}

The Binary Object Archive (BOA) is MONTE's primary data management system. Most MONTE classes that define concrete objects (for instance, M. Gm which defines the standard gravitational parameter for a natural body or M.FiniteBurn which

1. All MONTE code in this paper is current as of the v121 delivery.

2. Saturn Orbit Insertion was a spacecraft maneuver that occurred as Cassini approached Saturn. It changed the course of the spacecraft so that instead of flying past Saturn, it captured into orbit around the planet. defines a spacecraft burn) are stored in BOA, and accessed by MONTE's astrodynamic functions from BOA.

BOA is based on the binary XDR data format, which allows data to be written-to and read-from binary on different operating systems and using different transport layers (e.g. you can read and write locally to your hard disk, or over a network connection).

The role that BOA plays in MONTE can perhaps be best understood as "defining the universe" on which MONTE's astrodynamic tools operate. In our example, we populated our "model universe" (e.g. our BOA database) with time systems, natural body data, a planetary ephemeris, the Cassini spacecraft trajectory, etc. We then asked MONTE's trajectory manager (an astrodynamic tool) to examine this particular universe and return the state of Cassini with respect to Saturn.

\section{Default Data}

A standard MONTE installation comes with a collection of predefined, publicly available astrodynamic datasets (the "default data depot"). These can be accessed and loaded into a BOA database via MONTE's default data loader (mpy.io.data) and serve to help an analyst get a "model universe" up and running quickly.

\section{Time and Units}

In the astrodynamic community there are multiple time systems used to describe the dynamics of a spacecraft and to specify the time of an observation. While necessary, multiple systems for specifying time can add considerable complexity to software. In MONTE, time is encapsulated in the M.Epoch class, which supports time definition in the TDB, TT, TAI, GPS, UTC, and UT1 systems. This class handles the problem of transforming times between different frames thereby allowing the user to specify times in the most convenient form for their application.

MONTE's unit system supports the notions of time, length, mass, and angle. It has implemented operator overloading to allow unit arithmetic, e.g. dividing a unit length by a unit time results in unit velocity. Most functions that accept unit-quantities also check their inputs for correctness, so supplying a unit length to a function that expects unit time will raise an exception.

\section{Trajectories}

MONTE models spacecraft and natural body trajectories in a number of underlying formats; most of the differences involve how many data points along the trajectory are stored, and how to interpolate between these points. In addition, MONTE provides conversion routines which allow some external trajectory formats to be read and written (including NAIF "bsp" files and international "oem" files).

The M.TrajSet class is MONTE's trajectory manager, and is responsible for coordinating state requests between all of the trajectories loaded into a given BOA database. It has access to the coordinate frame system (described in the next section) allowing it to make coordinate frame rotations when doing state queries. In fact, most coordinate frame rotations in MONTE are accomplished by simply requesting a state from M.TrajSet in the desired frame.

The general steps for building and using trajectories in MONTE are illustrated in Figure 2.

\section{Coordinate Frames}

The MONTE trajectory and coordinate frame systems are very analogous and have a tight integration that enables powerful state 


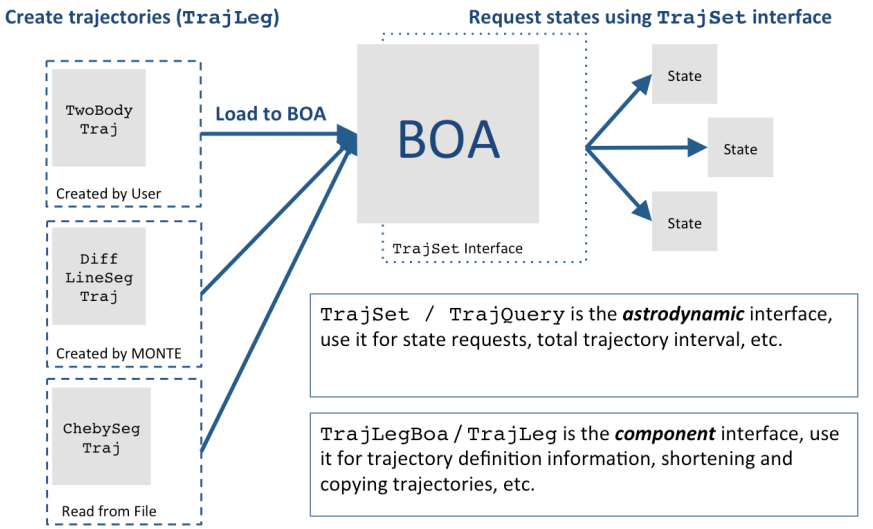

Fig. 2: Dataflow through MONTE's trajectory system

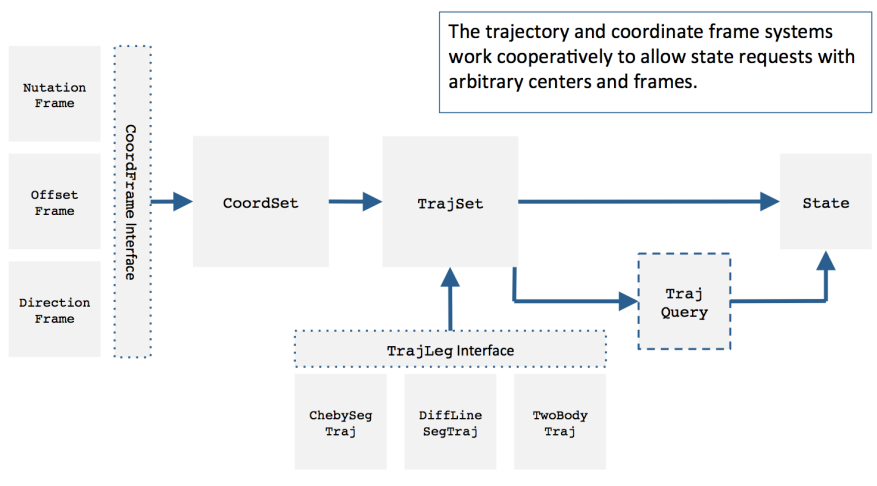

Fig. 3: Cooperation between MONTE's trajectory and coordinate frame systems

requests. Figure 3 illustrates these similarities and how the two systems are integrated.

MONTE models coordinate frames in a number of underlying formats and provides conversion routines which allow some external coordinate frame formats to be read and written (including NAIF "ck" files).

\section{Event Finding}

MONTE allows a user to search through astrodynamic relationships in a given BOA database in pursuit of particular events. For instance, the M.AltitudeEvent class allows a user to search for when a spacecraft is within a certain altitude range from another body.

\section{Numerical Integration}

MONTE provides a framework for numerically integrating spacecraft and natural body trajectories, subject to a set of force models such as gravity, solar radiation pressure, atmospheric drag, etc. The resulting trajectory has the Cartesian position and velocity of the body over time, and optionally the partial derivatives of state parameters with respect to parameters in the force models. A walkthrough of setting up MONTE's numerical integration system for a simple gravitational propagation is shown in Figure 4.

In addition to trajectories, MONTE also allows numerical integration of mass (for instance due to burning of propellant), coordinate frames (rigid body dynamics), time (relativistic time transformations) and user-defined ordinary differential equations.

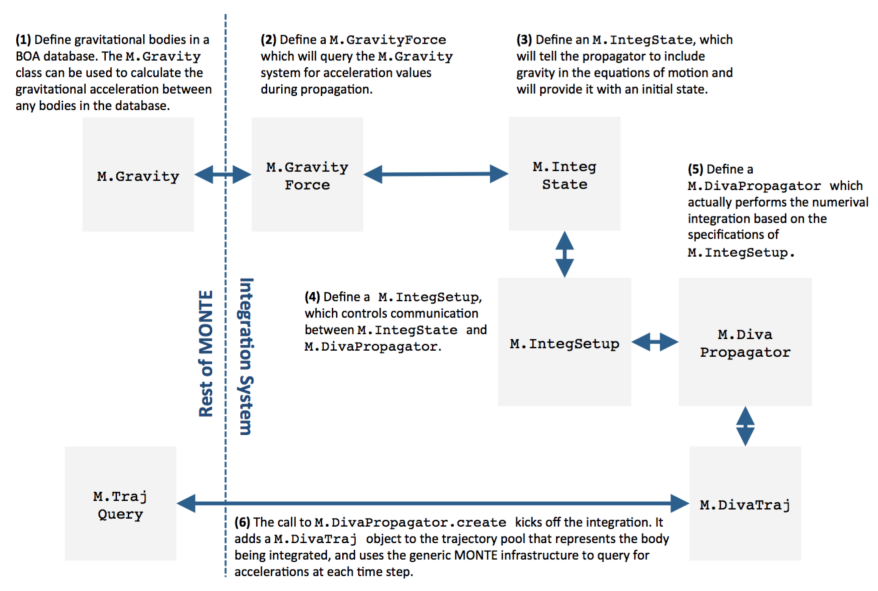

Fig. 4: Overview of MONTE's numerical integration system.

\section{Parameters and Partial Derivatives}

MONTE's parameter system supports the calculation of partial derivatives for astrodynamic variables, which can then be used in optimization and estimation. Every variable that belongs to the parameter system is responsible for not only calculating its value, but also its partial derivative with respect to any other parameters. These partial derivatives are contained in a special set of classes that employ operator overloading to correctly combine partial derivatives under various mathematical operations. [Smi16]

\section{Example: Exploring bodies in motion}

Generally, MONTE is scripted or assembled into custom applications that solve complex end-user problems. However, it is also useful as an off-the-cuff tool to explore astrodynamic relationships as we will see in the narrated example below.

For this example, we will explore the Voyager 2 trajectory. We will identify the time and distance of the Uranus planetary encounter, and also find the time periods where Voyager 2 was in line with the sun. Along the way we will highlight various aspects of MONTE's core systems. Also, if our exploration happens to turn up anything interesting (it will), we will take some time to investigate what we find.

\section{Voyager 2 Trajectory}

We begin by specifying the model of the solar system during Voyager's mission. This is done by creating a BOA database and loading the default data sets for planetary ephemerides (the trajectories of all the planets in the solar system), coordinate frames, and body parameters like mass and shape. We will also load in our Voyager 2 trajectory. ${ }^{3}$

3. JPL hosts two excellent websites for accessing trajectory data for natural solar system bodies and deep-space probes. The Horizons website (http //ssd.jpl.nasa.gov/horizons.cgi) is maintained by JPL's Solar System Dynamics group and has an expansive and powerful webapp for getting ephemerides in a variety of formats. The Navigation and Ancillary Data Facility (NAIF) at JPL hosts the navigation section of NASA's Planetary Database System. At its website (http://naif.jpl.nasa.gov/naif/data.html), you will find a host of downloadable binary navigation files, which can be used with the SPICE toolkit, and of course, with MONTE.

For the following examples, we will be using the Voyager 2 spacecraft trajectory, which can be downloaded at http://naif.jpl.nasa.gov/pub/ naif/VOYAGER/kernels/spk/. The file name at the time of this writing is "voyager_2.ST+1992_m05208u.merged.bsp", which we will shorten to just "voyager2.bsp" for ease of use. 
In [1]: import Monte as M

In [2]: import mpy.io.data as defaultData

In [3]: boa $=$ M. BoaLoad ()

In [4]: defaultData.loadinto( boa,

...: ["ephem/planet/de405", "frame", "body"] )

In [5]: boa.load( "voyager2.bsp" )

The trajectories of Voyager and the natural bodies of the solar system are coordinated by the trajectory manager (M.TrajSet) that is supplied by BOA we just created. We can retrieve the trajectory manager using its BOA accessor M. TrajSetBoa. Every object that resides in BOA has an accessor (often named M. ClassNameBoa) that allows it to be read to and from the database. Once in hand, we can list all the trajectories that are on the BOA using the M. TrajSet. getAll method.

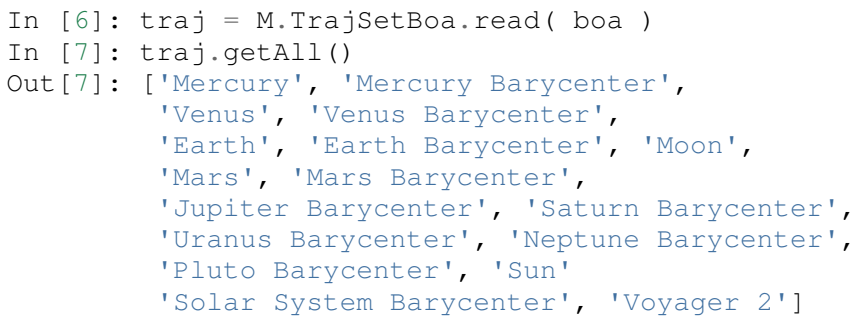

The list of bodies returned by M.TrajSet.getAll confirms that we have successfully loaded our solar system and spacecraft. We continue our analysis by checking the span of the Voyager 2 trajectory, e.g. the interval over which we have data, using the M.TrajSet.totalinterval method. Note that if the trajectory has been updated at the NAIF PDS website, the exact span you get may be different than what is listed below.

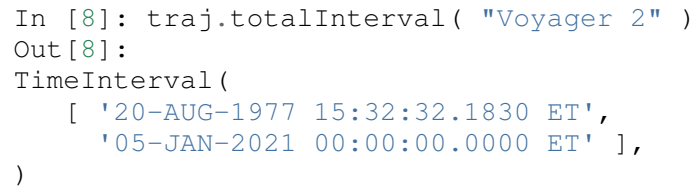

The Voyager 2 trajectory starts just after launch in 1977, extends through the present, and has predictions out into the future. We can use the trajectory manager to request states at any time in this window. For instance, we can find the distance of Voyager 2 from Earth right now. The M.Epoch. now static method returns the current time and this can be passed to the trajectory manager to request the state of Voyager 2 with respect to Earth.

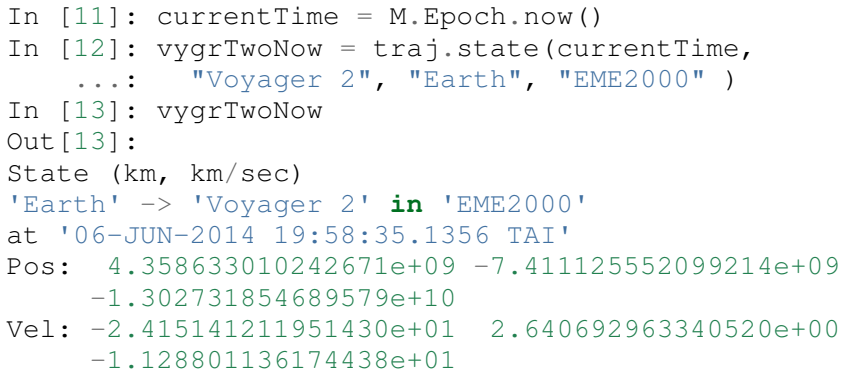

We used the M.TrajSet.state method to perform our query, which required us to specify the time, target body, reference body, and coordinate frame for the return state. Because M. TrajSet has a global view of all the trajectories in our BOA, we can request states with respect to any body for which we have a trajectory, for instance Venus or Neptune.

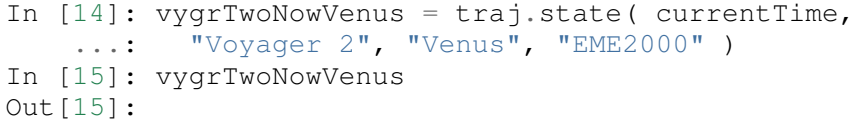

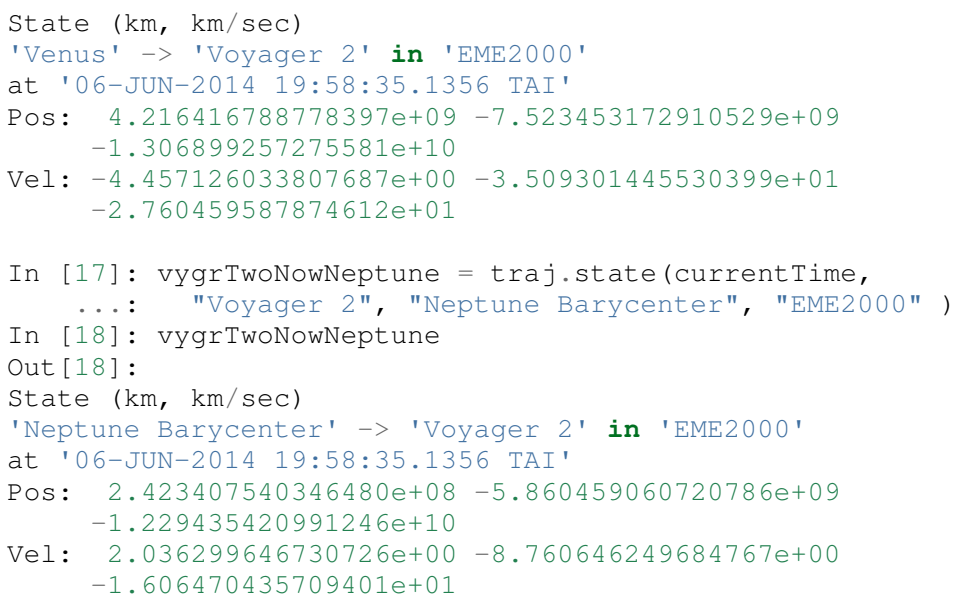

The M.TrajSet.state method returns an M.State object. M.State captures the relative position, velocity and acceleration (or some subset) of one body with respect to another at a given time. It has a number of methods that help with extracting and transforming the information it contains. For instance, we can find the distance from Earth to Voyager 2 like this.

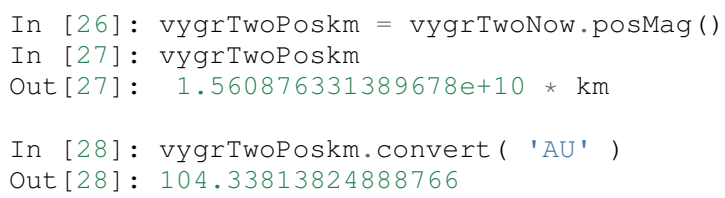

When reading states from a trajectory you are often interested in making repeated calls for the same body and center but at different times. M.TrajSet works fine for this application, but if the target and center bodies don't change on repeated calls, some optimizations can be made for better performance. The M. TrajQuery class is provided for this use case, and can be thought of as simply a special case of M.TrajSet where the body and center are fixed for every call.

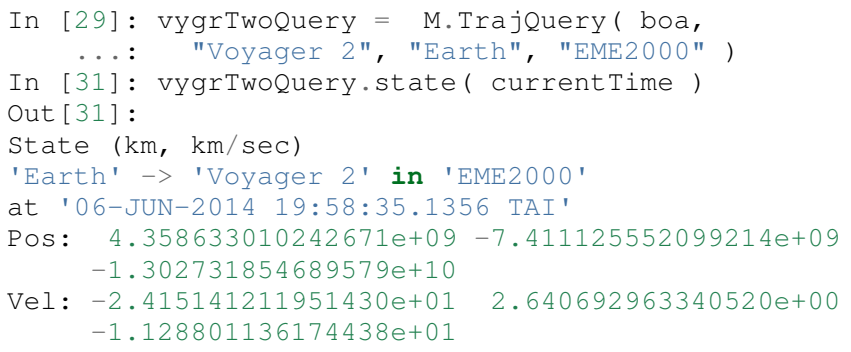

This can be useful when you are sampling states from a trajectory, for instance, to create a plot of an orbit.

\section{Uranus Encounter}

We said earlier that M.TrajSet and M.CoordSet, in their roles as manager classes, have a global view of the trajectory and coordinate systems. This high-level perspective allows them to work with the relationships between different bodies and frames, a capability we have so far used to get relative states between bodies. However, there are certain specific relationships between bodies and frames that can be of particular interest to an analyst. For instance, identifying the time at which two bodies achieve their closest approach (periapse) and the magnitude of that minimum distance can be an important astrodynamic metric. MONTE provides tools for searching through various relationship-spaces and identifying some of these key events. The M.EventSpec 
set of classes allow us to define a particular event type then search through the requisite relationships to identify specific occurrences. The M.Event class is used to report the relevant data associated with an occurrence.

Continuing the example, we will use M. ApsisEvent (which is a specific type of M.EventSpec) to find the precise time and distance of Voyager 2's closest approach with Uranus.

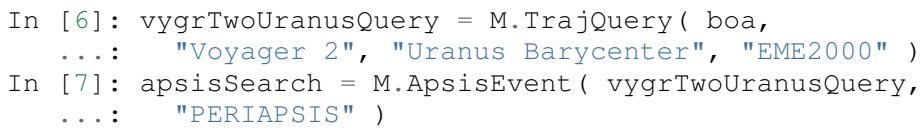

M.ApsisEvent takes as its first argument an M.TrajQuery object that is configured to return the state of our target body with respect to the desired center (in this case, Voyager 2 with respect to Uranus). The second argument specifies what type of apsis we are looking for; this can be "PERIAPSIS", "APOAPSIS", or the catch-all "ANY". Once the event type is defined, the M.ApsisEvent. search method can be called to perform the search and locate the apses. To call this method we need to provide a time interval to search over and a search step size.

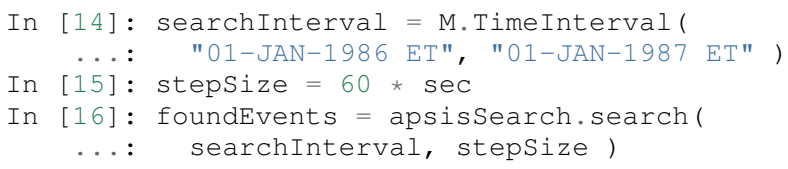

The result of the search, which we have saved in the variable foundEvents, is an M.Event Set container class. This container has all the events found matching our specification in the search window. M.Event Set has a number of useful methods for sorting, filtering and returning events. In this case there should only be one event returned since there was only one closest approach of Voyager 2 to Uranus. We can read out this event by indexing into the M.Event Set.

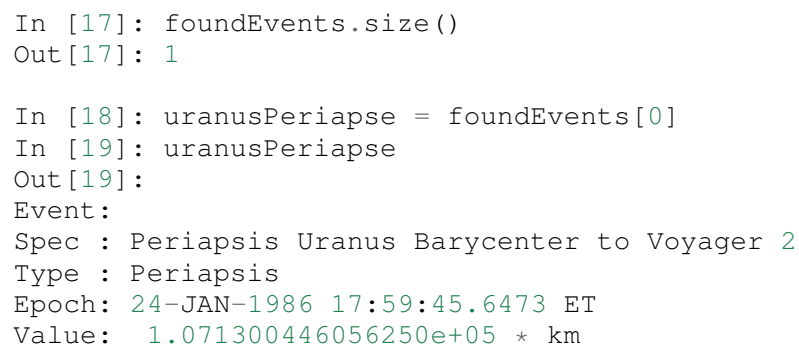

Another relationship which can play a significant role in deep space missions is the angular offset between the Earth-Sun line and Earth-Spacecraft line (often referred to as the Sun-Earth-Probe (SEP) angle). At low SEP values, the spacecraft appears very close to the Sun from the vantage of Earth, requiring radio transmissions from Earth to pass through the near-solar environment before reaching the spacecraft. Flight projects avoid critical mission operations during these times because the highly-charged solar atmosphere can interfere with radio signals.

We can set up an event search to find periods of low-SEP for Voyager 2, from mission start through the end of our trajectory data, using the M. AngleEvent event specification class.

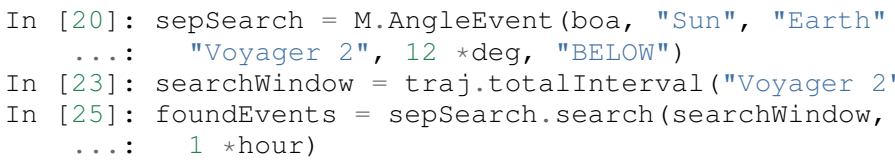

We constructed our M. AngleEvent by defining the Sun-EarthProbe angle using the Sun for body one, the Earth as the vertex, and Voyager 2 as body two. Twelve degrees was set as the threshhold defining conjunction, and the "BELOW" qualifier was used to instruct the search to return times when the SEP angle was below this threshold.

The search again returned an M.Event Set, which we can use to get information about the number of events found and the maximum / minumum times Voyager 2 spent in conjunction.

In [26]: foundEvents.size()

Out [26]: 15

In [52]: foundEvents.maxinterval ()

Out [52]:

Event:

Type : Angle below 1.200000000000000e+01 * deg

Begin: 28-JUN-1978 07:34:09.7021 ET

End : 03-AUG-1978 05:22:28.3997 ET

Value: $1.199999999999977 e+01 *$ deg

In [53]: foundEvents.minInterval()

Out [53]:

Event:

Type : Angle below 1.200000000000000e+01 * deg

Begin: 31-DEC-1992 09:35:21.3322 ET

End : 07-JAN-1993 21:30:07.6066 ET

Value: $1.199999999999999 e+01$ * deg

We can loop through all the events found in our search using Python iterator syntax, and print out the time periods of each found low-SEP region.

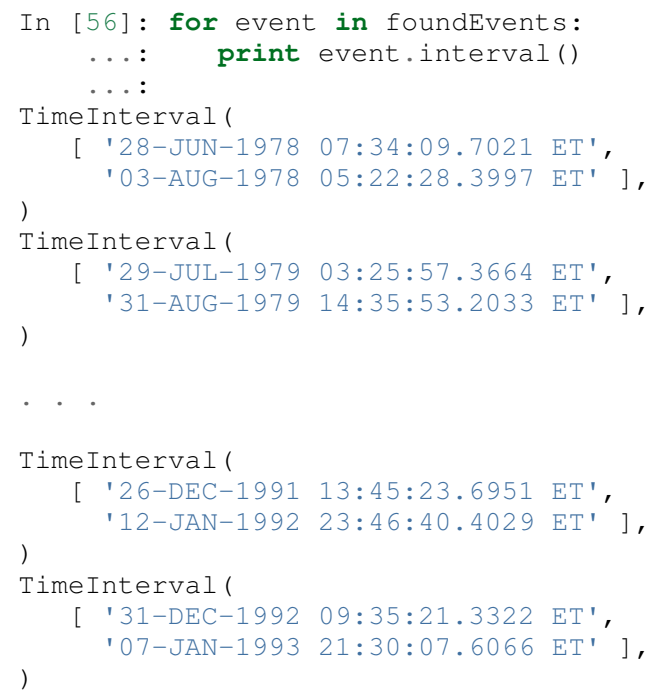

As we can see, low-SEP periods occur on a near-yearly basis. This makes sense because as the Earth makes a complete revolution around the Sun, there is bound to be a period of time when the Sun falls in the line-of-sight of Voyager 2. Curiously though, the last low-SEP region found was in the winter of 1992. After this time, the Sun no longer obscures the Earth's view of Voyager 2 at all! Evidently, Voyager 2s trajectory changed in a way that disrupted this the annual low-SEP viewing geometry dynamic.

If Voyager 2 were to somehow leave the plane of the solarsystem, the Earth would have a constant unobstructed view of the spacecraft permanently. We can investigate this theory by looking ) at the distance of Voyager 2 from the solar system ecliptic plane. We do this by setting up a trajectory query to return the state of Voyager 2 with respect to the Sun in EMO2000 coordinates (the EMO2000 coordinate frame measures $\mathrm{Z}$ with respect to the solar system plane). The Z-component of the position vector will then 


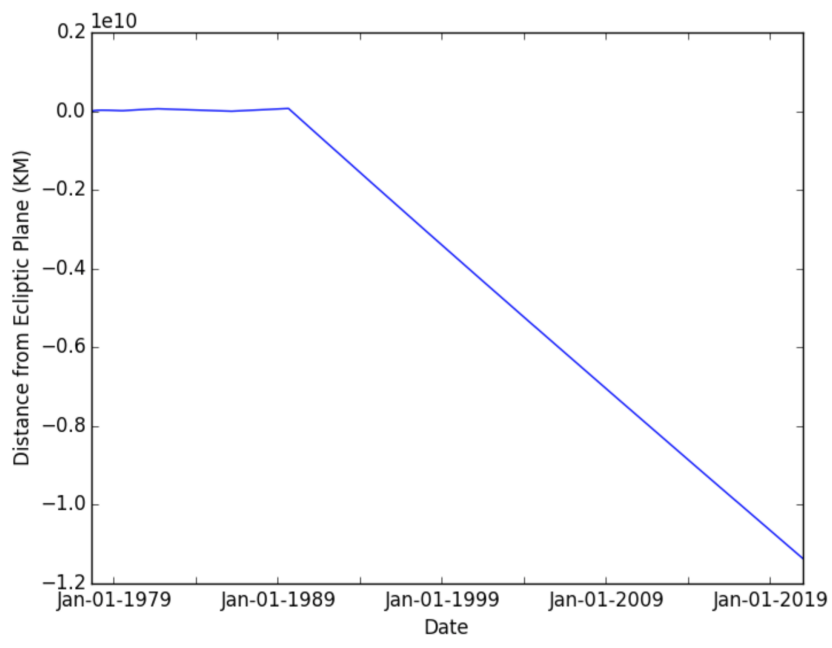

Fig. 5: Distance in kilometers of Voyager 2 from the solar system ecliptic plane.

yield the offset from the ecliptic plane. We will plot this distance over the course of the Voyager 2 mission to see how this distance evolves.

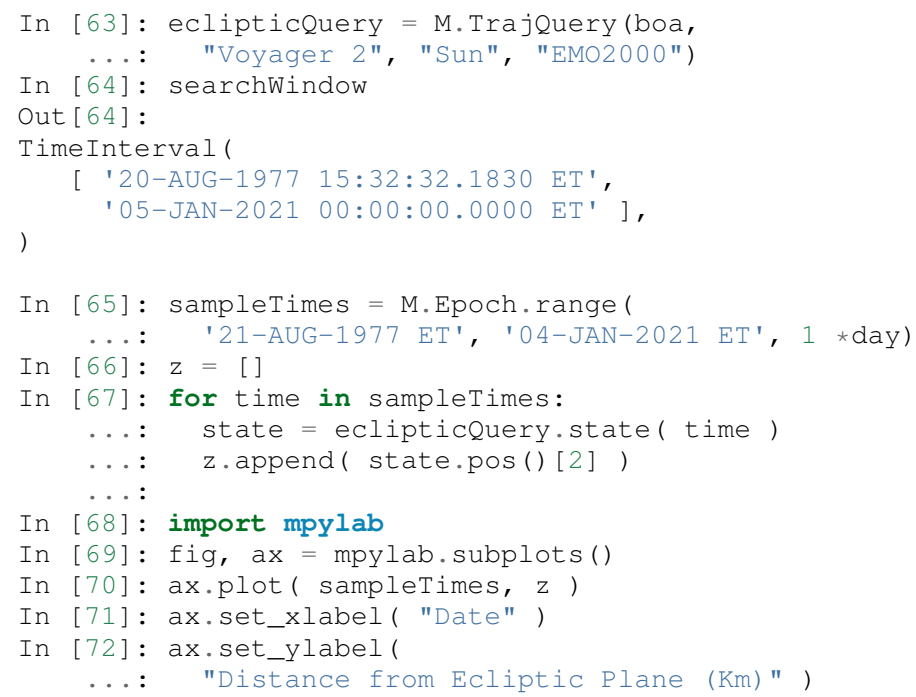

The generated plot is shown in Figure 5.

It appears that something happened in 1989 to cause Voyager 2 to depart from the ecliptic plane. A quick glance at the Wikipedia page for Voyager 2 confirms this, and reveals the cause of this departure.

Voyager 2's closest approach to Neptune occurred on August 25, 1989 ... Since the plane of the orbit of Triton is tilted significantly with respect to the plane of the ecliptic, through mid-course corrections, Voyager 2 was directed into a path several thousand miles over the north pole of Neptune ... The net and final effect on the trajectory of Voyager 2 was to bend its trajectory south below the plane of the ecliptic by about 30 degrees.

\section{Conclusion}

MONTE is one of the most powerful astrodynamic computing libraries in the world. It has been extensively tested and verified by flying actual spacecraft to destinations in the solar system. It is a compelling platform for anyone doing aerospace related computation, especially for those who love working with the Python language.

\section{Acknowledgements}

This work was carried out at the Jet Propulsion Laboratory, California Institute of Technology, under a contract with the National Aeronautics and Space Administration.

\section{RefERENCES}

[Moy71] T. Moyer, Mathematical Formulation of the Double-Precision Orbit Determination Program (DPODP), TR 32-1527 Jet Propulsion Laboratory, Pasadena 1971.

[Moy03] T. Moyer, Formulation for Observed and Computed Values of Deep Space Network Data Types for Navigation, John-Wiley \& Sons, Inc. Hoboken, Jew Jersey, 2003.

[Eke05] J. Ekelund, History of the ODP at JPL, Internal Document, Jet Propulsion Laboratory, Pasadena 2005.

[Smi16] J. Smith, Distributed Parameter System for Optimization and Filtering in Astrodynamic Software, 26th AAS/AIAA Spaceflight Mechanics Meeting 2016 proceedings, Napa, CA

[Eva16] S. Evans, MONTE: The Next Generation of Mission Design \& Navigation Software, The 6th International Conference on Astrodynamics Tools and Techniques (ICATT) proceedings 2016, Darmstadt, Germany. 